\title{
Infrared Emission Spectrum of Calcium ${ }^{1}$
}

\author{
Curtis J. Humphreys
}

\begin{abstract}
The spectrum of neutral calcium excited in alternating-current and direct-current ares, using carbon electrodes cored with $\mathrm{CaO}$, has been observed with high resolution, in the interval between 12816 and 22655 Angstroms. A total of 36 lines has been observed, of which all but one have been classified. The long-sought term, $3 d^{2}{ }^{3} \mathrm{~F}$, has been found and established on the basis of two complete multiplets, $3 d \cdot 4 p^{3} \mathrm{~F}^{\circ}-3 d^{2}{ }^{3} \mathrm{~F}$, and $3 d \cdot 4 p^{3} \mathrm{D}^{\circ}-3 d^{2}{ }^{3} \mathrm{~F}$, and two intersystem lines $3 d \cdot 4 p^{1} \mathrm{D}^{\circ}-3 d^{2}{ }^{3} \mathrm{~F}_{2,3}$. Most of the infrared energy is radiated in the multiplet $4 p^{3} \mathrm{P}^{\circ}-3 d^{3} \mathrm{D}$. These lines are suggested for use as wavelength standards for the 2-micron region.
\end{abstract}

\section{Introduction}

The study of infrared atomic emission spectra has been given new impetus as a result of the utilization of photo-conducting cells in conjunction with high-resolution grating spectrometers for observation in the region just beyond the limit of response of infrared-sensitive photographic emulsions. Previous to this recent renewal of activity there had been an almost complete cessation of work on infrared atomic emission spectra for a period of about 25 years, following the work of Paschen and his pupils. The explanation lies mainly in the relative insensitivity of thermal detectors, such as thermopiles, bolometers, and radiometers, as compared with photographic emulsions in regions where they could be used. In order to observe infrared emission spectra at all, it was necessary to work with optical slits so wide that the precision of wavelength determinations was insufficient to distinguish between real and fortuitous regularities, with the result that the observational data were not of much value for term analyses. It was also impossible to observe lines other than those of very high intensity, thus providing an insufficient number of wavelengths for use in establishing regularities.

The use of photoconducting detectors now provides essentially the same advantages as photography, as regards both volume of attainable data and precision of observations, particularly for the region between 1 and $3 \mu$ where lead sulfide cells may be used. It is obviously possible to observe infrared lines for most if not all atoms in this region. Many of these lines can be predicted in position on the basis of term analyses already made with varying degrees of completeness. Three criteria are suggested as the basis of selection of spectra for study; first, gaps in the term system that can be filled in only by finding regularities involving infrared data; second, astrophysical interest, such as questions of identification of elements in the solar spectrum on the basis of excitation in the laboratory, and, finally, usefulness in providing wavelength standards that may assist in the measurement of wavelengths in other spectra, either atomic or molecular.

Observations have been completed or are now in progress on several infrared emission spectra in the

1 Reported, in part, at the meeting of the Optical Society of America, Washington, D. C., March 1, 1951.
Radiometry Laboratory. Ca I has been selected for the first detailed report because it is probably the most conspicuous example of a spectrum in urgent need of observation on the basis of all three suggested criteria.

\section{Energy Levels of Ca I}

$\mathrm{Ca} \mathrm{I}$ is an example of a relatively simple spectrum, the term system comprising singlets and triplets, arising from two valence electrons outside of closed shells. Table 1 lists the electron configurations with designations of predicted terms appropriate to each. A more detailed table is to be found on page XXXV of Bureau Circular 467 [1]. ${ }^{2}$ The numerical values of all energy levels of Ca I previously known are listed in the same book, together with references to the sources of the material.

TABLE 1. Electron configurations and terms of Ca I

\begin{tabular}{|c|c|}
\hline Configuration & Predicted terms \\
\hline $\begin{array}{l}4 s^{2} \\
4 s\left({ }^{2} \mathbf{S}\right) 5 s \\
4 s\left({ }^{2} \mathbf{S}\right) 4 p \\
4 s\left({ }^{2} \mathbf{S}\right) 3 d \\
4 s\left({ }^{2}\right) 4 f \\
3 d\left({ }^{2} \mathrm{D}\right) 4 p \\
4 p^{2} \\
3 d^{2}\end{array}$ & $\begin{array}{ll}{ }^{1} \mathrm{~S} & \\
{ }^{1} \mathrm{~S} & ,{ }^{3} \mathrm{~S} \\
{ }^{1} \mathrm{P}^{\circ} & ,{ }^{3} \mathrm{P}^{\circ} \\
{ }^{1} \mathrm{D} & ,{ }^{3} \mathrm{D} \\
{ }^{1} \mathrm{~F}^{\circ} & ,{ }^{3} \mathrm{~F}^{\circ} \\
{ }^{1}(\mathrm{P}, \mathrm{D}, \mathrm{F})^{\circ},{ }^{3}(\mathrm{P}, \mathrm{D}, \mathrm{F})^{\circ} \\
{ }^{1}(\mathrm{~S}, \mathrm{D}) & ,{ }^{3} \mathrm{P} \\
{ }^{1}(\mathrm{~S}, \mathrm{D}, \mathrm{G}) & ,{ }^{3}(\mathrm{P}, \mathrm{F})\end{array}$ \\
\hline
\end{tabular}

The regular series, resulting from the displacement of one electron from the normal $4 s^{2}$ configuration to successively higher states, have been known almost from the first discovery of spectral regularities. The information is summarized in the well-known monographs by Paschen and Götze [2] and by A. Fowler [3]. It was pointed out in 1925 by Russell and Saunders [4] that, in Ca I and other alkalineearth spectra, features of these spectra not accounted for by regular series could be explained as transitions between terms arising from electron configurations in which both electrons are displaced from the normal configuration. This paper, which preceded the formal presentation of the Hund theory [5], served in a sense as an introduction to the theory of complex spectra.

\footnotetext{
2 Figures in brackets indicate the literature references at the end of this paper.
} 
$+13711 \mathrm{~cm}^{-1} \ldots \ldots \ldots \ldots$ Limit, $3 \mathrm{~d}^{2} \mathrm{D}_{2 \frac{1}{2}}$, CaII
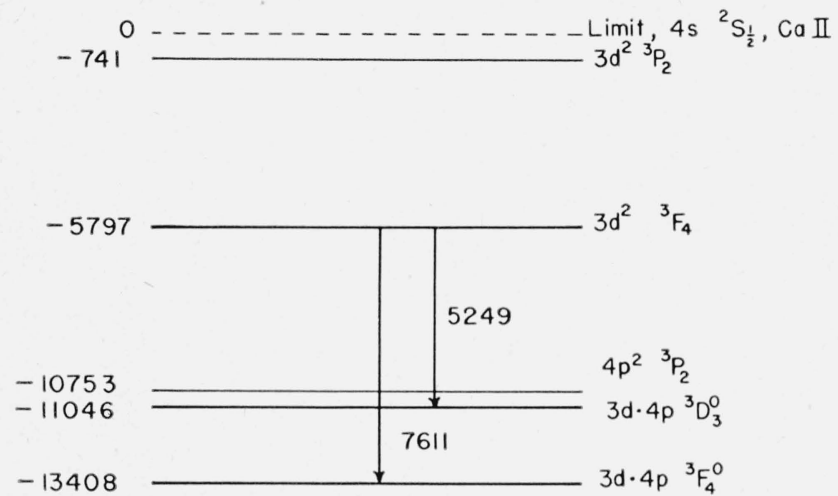

Figure 1. Partial term diagram of $\mathrm{Ca} \mathrm{I}$.

In spite of extensive efforts that had been made to complete the analysis of CaI, there remained, previous to the observations reported in this paper, a conspicuous gap in the term array, namely, that, with the exception of ${ }^{3} \mathrm{P}$, all terms from the $3 d^{2}$ configuration were missing. The $3 d^{2}{ }^{3} \mathrm{P}$ term is abnormally high, possibly because of a perturbation by $4 p^{2}{ }^{3} \mathrm{P}$, another term of even parity. This perturbation has the effect of a repulsion, producing a separation of terms that would otherwise be at about the same elevation. Combinations of $3 d^{2}{ }^{3} \mathrm{P}$ with lower odd terms yield photographically observable lines, permitting evaluation of the term. There is no such perturbing term to affect the position of $3 d^{2}{ }^{4} \mathrm{~F}$. Its location was predicted by $\mathrm{H}$. N. Russell in accordance with the following considerations, communicated privately. The higher members of this series, converging to the $3 d^{2} \mathrm{D}$ limit in Ca II, are known, permitting evaluation of total quantum numbers and the values of $\Delta n$. Assuming $\Delta n$ to be a little greater than 1 for the first two members of the ${ }^{3} \mathrm{~F}$ series, the absolute value of $3 d^{2}{ }^{3} \mathrm{~F}_{4}$ was predicted to be $20700 \mathrm{~cm}^{-1}$ with respect to its own limit. Taking into account the elevation of this limit, $13711 \mathrm{~cm}^{-1}$ above $4 s^{2} \mathrm{~S}$ in Ca II, the ionization limit of the regular series, the ${ }^{3} \mathrm{~F}_{4}$ level is expected about $7000 \mathrm{~cm}^{-1}$ below the usual ionization limit in Ca I. Translating this into the usual system, as employed in Circular 467 [1], in which the ground level is given the value zero, the predicted value of $3 d^{2}{ }^{3} \mathrm{~F}_{4}$ becomes approximately 42300 . This is accomplished by subtracting 7000 from 49304.80 , the value of the first ionization limit. Actually, $3 d^{2} \mathrm{~F}_{4}$ was found to be 43508.11 , or $5797 \mathrm{~cm}^{-1}$ below this limit, in excellent agreement with the prediction, considering the uncertainty in the estimate of $\Delta n$. Figure 1 is introduced to assist in visualizing the relative positions of the terms that are of significance in determining the location of $3 d^{2}{ }^{3} \mathrm{~F}$. For simplicity, only a single level, that of highest $J$-value, is drawn in the position of each term. The displacements indicated are with reference to the limit of the regular series, that is the $4 s^{2} \mathrm{~S}$ ground level of Ca II. The diagram includes the $3 d 4 p^{3} \mathrm{D}_{3}^{\circ}$ and ${ }^{3} \mathrm{~F}_{4}^{\circ}$ levels, representing the terms that give the strongest combinations with $3 d^{2}{ }^{3} \mathrm{~F}$. The experimental material completely establishing all the levels comprising this term will be presented in a later section of this paper.

\section{Observations}

The development of photoconducting detectors and of amplifying and recording equipment has now advanced sufficiently that energy detection no longer represents the principal difficulty in infrared observation. The high-resolution spectrometer recently completed and put in use in the radiometry laboratory of the Bureau has been described previously [6]. A 15,000 lines-per-inch plane grating, 71/2 in. wide, supplied by Johns Hopkins University, is used for observations in the region between 1 and $3 \mu$. Where one must obtain the record of the spectrum on a paper chart by continuous scanning, the most serious problem in observing line spectra is to find a source combining sufficient excitation of highenergy states with operation steady enough to give a smooth development of line profiles, permitting precise location of the peaks for wavelength determinations. An enclosed source, such as a Geissler tube, or the various metal vapor lamps utilizing relatively volatile metals, for example, mercury, cadmium, or the alkalis, is ideal for such use. Calcium unfortunately does not lend itself readily to such use because it has a fairly high melting point and combines readily with the glass enclosures at elevated temperatures.

The reasonably smooth operation of open flame alternating-current arcs, using carbons cored with various salts in order to increase emission in selected spectral regions, suggested that a satisfactory calcium source might be developed by using this principle. Unfortunately, no commercially prepared carbons cored with calcium salts were available. The problem was, however, brought to the attention of the National Carbon Co., and this company generously offered to supply experimental carbons cored with calcium salts and other metallic compounds to be used in other investigations now on our program. A supply of these carbons has been received, but so far has been used only to a limited extent. Most of the observations reported in this paper had been completed before the arrival of these carbons. Inasmuch as subsequent work on this and other elements requiring similar sources will probably utilize the electrodes supplied by the National Carbon Co., an extremely brief report will be given of the preparation of cored carbons in the laboratory and of methods of use. 
Fadometer carbons one-half in. in diameter were used in these experiments. The arc was assembled with one plain electrode and one cored with a salt of the element under study. Operation of the arc on alternating-current supply was abandoned after early trials when it was found that satisfactorily smooth operation could be obtained on direct current, and that, as might be expected, greatly improved excitation resulted when the cored carbon was made positive. Most of the cored electrodes were made by drilling a $3 / 16$-in. opening in a plain carbon along the axis and packing with a mixture of calcium oxide and carbon dust. The calcium oxide was the most satisfactory of a number of compounds tried, but, in view of the development work by the National Carbon Co., no exhaustive study was made. The handoperated arc was powered by the $220-\mathrm{v}$ direct-current supply available in the laboratory, ballasted by sufficient resistance to maintain the current in the range between 15 and $25 \mathrm{amp}$. This was close to the maximum operating load of the circuits as assembled. It did not necessarily represent the optimum conditions for smooth arc operation or excitation of desired energy levels. However, operating conditions were adequate to permit attainment of the primary objectives of the experiments.

One troublesome experimental difficulty arose in connection with the operation of the carbon ares. The entire spectral region extending from about 1.1 to beyond $1.6 \mu$, except for a short gap near $1.3 \mu$, is covered by two sets of band progressions of the CN system, corresponding to two vibrational transitions. Wavelengths of the $(0,0)$ band heads were reported by Kiess [7] from photographic observations, and the entire infrared system was observed with relatively low dispersion by Herzberg and Phillips [8]. These bands were completely resolved in these experinents and will be reported in a subsequent publication. This band structure is so intense and closely packed that other spectral structures cannot be distinguished if they fall within these bands. One of the multiplets involving the newly found ${ }^{3} \mathrm{~F}$ term, $3 d\left({ }^{2} \mathrm{D}\right) 4 p^{3} \mathrm{~F}^{\circ}-$ $3 d^{2}{ }^{3} \mathbf{F}$, is within the gap between the two bands. There is, however, enough weak band structure to prevent observation of the satellite lines. It was found possible to eliminate most of the band structure by using an enclosed water-cooled arc, circulating helium through the arc chamber at a rapid rate. To offset the suppression of the excitation accompanying the introduction of helium, the positive carbon was cored with a slug of calcium metal replacing the $\mathrm{CaO}$. This permitted the tracing of satisfactory records over short ranges. The calcium is used up rapidly in the enclosed arc and does not operate in controllable fashion in the open arc because of extremely rapid conversion to oxide. The enclosed arc, used for many years in the Spectroscopy Laboratory, was originally designed by H. D. Curtis [9], and kindly loaned for these experiments by William F. Meggers.

The optical system in front of the entrance slit of the spectrometer was set up in order to satisfy two conditions in addition to full illumination of the collimating mirror. These were elimination of continu-

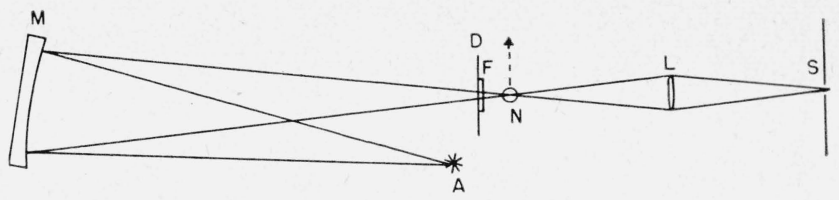

FiguRE 2. Arrangement for projecting image of source upon slit of spectrometer.

A, Arc lamp; N, neon tube; M, concave mirror; D, diaphragm with horizontal slot; F, filter; L, quartz lens; S, spectrometer slit.

ous emission from the electrodes and provision for introduction of a source of standard wavelengths. The arrangement of components is sketched in figure 2, as seen from above. A quartz lens and a concave mirror were lined up on the optic axis. These were at such distances that a slightly magnified image of the source, set up slightly off axis, was formed by the concave mirror on the axis at a point such that a sharp image of the first image was projected onto the slit. A horizontal diaphragm was mounted between the lens and mirror, so that the images of the electrodes could be seen on the upper and lower jaws of the diaphragm with the arc stream between. The length of the arc and the diaphragm opening were adjusted to give the best operating conditions. In general, ares of about $6-\mathrm{mm}$ length gave the most satisfactory operation. A Corning 2540 filter was attached to the back of this diaphragm to eliminate higher orders of spectra. Actually, this diaphragmfilter combination was placed a little out of focus nearer the mirror. This permitted inserting the source of standard wavelengths at the common focus of the mirror and lens, making possible the use of wavelengths of any desired order. The Corning filter begins to transmit at about $0.9 \mu$. A neon discharge tube was used as a source of comparison spectra for determining the wavelengths of most of the observed lines, because second or third orders of the intense red lines happened to be most favorably located with reference to calcium lines. The neon tube was brought into position as required while the spectrum was being scanned. In a few instances, determined by the wavelengths to be measured, a krypton source was used.

The records used for wavelength determinations were run at the slowest scanning speed, approximately $16 \mathrm{~A}$ a minute at $20000 \mathrm{~A}$, insofar as operating conditions permitted. It was found possible to introduce standards close enough together in all instances that a linear interpolation could be used for reduction.

The shortest wavelength measured was of $12816 \mathrm{~A}$. This is barely within the range of photography. It is just at the beginning of the intense portion of the $(0,0) \mathrm{CN}$ band. For these reasons and also because the transitions especially sought had been found, it did not appear profitable to extend the measurements to shorter wavelengths. The spectrum was explored out to the limit of sensitivity of the lead sulfide cell near $27000 \mathrm{~A}$, but no first-order lines of appreciable intensity were found beyond $22655 \mathrm{~A}$. The $3 d^{2} \mathrm{D}-$ $4 p^{2} \mathrm{P}^{\circ}$ doublet of Ca II near $8600 \mathrm{~A}$ appeared with such intensity in the second and third orders that the 
lines were recorded even with the cutoff filters used to exclude higher orders.

\section{Discussion of Results}

The observation of the infrared spectrum of calcium was included in the very early work previously mentioned. Such observations were reported by Paschen [10], by Randall [11], and by Sandvik and Spence [12]. These observations were subject to the limitations in precision already mentioned, and, although most of the lines reported appear to be real, the levels represented in the transitions could be de- termined more precisely by other combinations in regions more favorable to observation. None of the lines involving combinations of the heretofore missing $3 d^{2}{ }^{3} \mathrm{~F}$ term was reported in any previous work. Having in mind that an entirely different order of precision of wavelength measurement has now been realized, a completely new description of the infrared spectrum has been prepared without attempting to associate any lines with probable counterparts from earlier work.

Descriptive data pertaining to 36 lines of $\mathrm{Ca} \mathrm{I}$ observed and measured in these experiments are compiled in table 2. These data comprise observed

TABLE 2. Description of $\mathrm{Ca} \mathrm{I}$ in the infrared region

[C, Probably confused by line at $16156.04 \mathrm{~A}$; u, Unresolved with respect to adjacent strong line

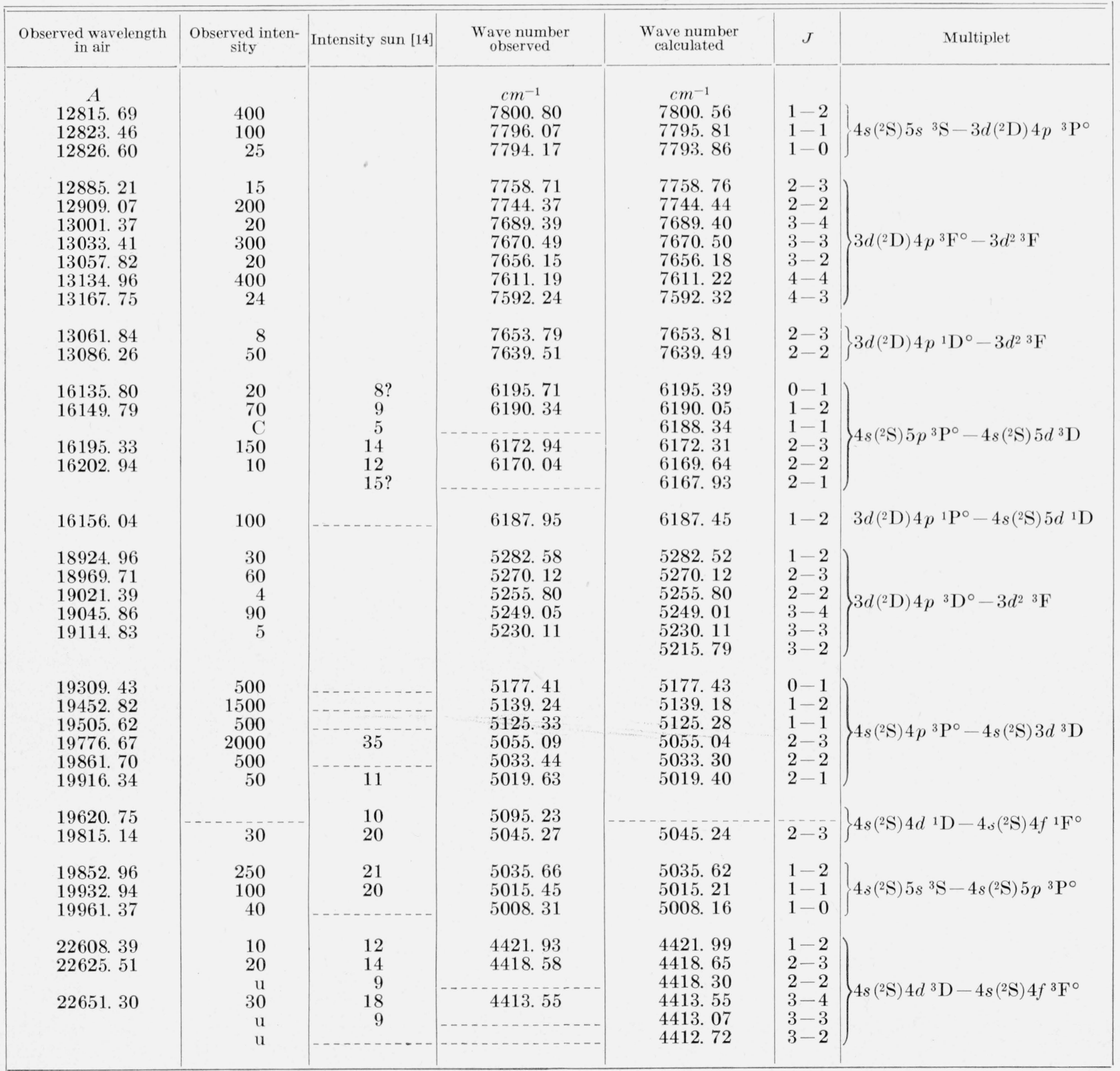


wavelengths at atmospheric pressure, corresponding wave numbers in vacuum, calculated wave numbers, intensity estimates, and classification where determined, as shown by multiplet designations. The reduction to wave numbers for wavelengths greater than $10000 \mathrm{~A}$ is accomplished by inversion of the Kayser "Tabelle der Schwingungszahlen", as suggested by Babcock [13] and applying the required correction. There is some uncertainty regarding the index of refraction of air for this region, but there is no evidence that this uncertainty introduces an error into wave-number reductions as large as the random errors inherent in the observational procedures. The wavelengths are carried out to seven figures in the table, that is to hundredths of angstroms. On the basis of experimental checking of known constant term differences, considerable confidence is felt in the sixth digit for all lines. The seventh is very uncertain but is retained for uniformity throughout the table, since it is regarded as probably significant for the best determined wavelengths. In most instances the listed wavelengths represent averages of from four to six measurements each on separate records. In a few cases ten or more measurements are included.

The indicated intensities may be regarded as semiquantitative. A precision technique for evaluating intensities has not been developed for the equipment in use, but such a development appears feasible. Intensity estimates are rendered somewhat uncertain for an arc source, because on the basis of repeated runs it appears to be subject to slow variation in energy output even when apparently steady. The relative intensities are much more reliable within multiplets than in the intercomparison of multiplets. Spectral sensitivity of the detector must be considered in any precise intercomparison over wide ranges. As is to be expected, the intensity distribution in multiplet patterns points to a very close realization of the $L S$-coupling scheme for Ca I.

One column of table 2 contains a listing of the intensities of lines in the solar spectrum observed by Goldberg, Mohler, Pierce, and McMath [14], where coincidences occur with the laboratory observations here reported. Calcium is one of the abundant metallic elements in the sun and is outstanding in the emission spectrum of the chromosphere and prominences. The laboratory observation and description of the infrared-calcium spectrum is expected to aid in the identification of calcium lines in this portion of the solar spectrum.

The most important result of this work, representing the attainment of the principal objective of the investigation, was the discovery of the multiplets comprising the combinations of $3 d^{2}{ }^{3} \mathrm{~F}$. The identification and interpretation of these multiplets are based on the observed fact that they involve combinations with the previously known levels from the $3 d .4 p$ configuration, also that such combinations are expected in these regions in accordance with the discussion of the level scheme already given. These multiplets are displayed in table 3 . The adopted

TABLe 3. Multiplet combinations of $3 d^{2}{ }^{3} \mathrm{~F}$ in $\mathrm{Ca} \mathrm{I}$

\begin{tabular}{|c|c|c|c|c|}
\hline & & $\begin{aligned} & 3 d^{2}{ }^{3} F_{4} \\
& \text { 43508. } 11\end{aligned}$ & 43489. ${ }^{3} F_{3}$ & $\begin{array}{c}{ }^{3} F_{2} \\
43474.89\end{array}$ \\
\hline $3 d \cdot 4 p^{3} \mathrm{~F}_{4}^{\circ}$ & 35896.890 & $\left\{\begin{array}{r}13134.96(400) \\
7611.19 \\
7611.22\end{array}\right.$ & $\begin{array}{r}13167.75(24) \\
7592.24 \\
7592.32\end{array}$ & \\
\hline${ }^{3} \mathrm{~F}_{3}^{\circ}$ & 35818. 712 & $\left\{\begin{array}{r}13001.37(20) \\
7689.39 \\
7689.40\end{array}\right.$ & $\begin{array}{r}13033.41(300) \\
7670.49 \\
7670.50\end{array}$ & $\begin{array}{r}\text { 13057. } 82(20) \\
7656.15 \\
7656.18\end{array}$ \\
\hline${ }^{3} \mathrm{~F}_{2}^{\circ}$ & 35730.450 & & $\left\{\begin{array}{r}12885.21 \\
7758.71 \\
7758.76\end{array}\right.$ & $\begin{array}{r}12909.07(200) \\
7744.37 \\
7744.44\end{array}$ \\
\hline $3 d \cdot 4 p^{1} \mathrm{D}_{2}^{\circ}$ & 35835. 400 & & $\left\{\begin{array}{c}13061.84(8) \\
7653.79 \\
7653.81\end{array}\right.$ & $\begin{array}{r}13086.26(50) \\
7639.51 \\
7639.49\end{array}$ \\
\hline $3 d .4 p^{3} \mathrm{D}_{3}^{\circ}$ & 38259. 102 & $\left\{\begin{array}{r}19045.86(90) \\
5249.05 \\
5249.01\end{array}\right.$ & $\begin{array}{r}19114.83(5) \\
5230.11 \\
5230.11\end{array}$ & 5215.79 \\
\hline${ }^{3} \mathrm{D}_{2}^{\circ}$ & 38219. 094 & & $\left\{\begin{array}{r}18969.71(60) \\
5270.12 \\
5270.12\end{array}\right.$ & $\begin{array}{r}19021.39(4) \\
5255.80 \\
5255.80\end{array}$ \\
\hline${ }^{3} \mathrm{D}_{1}^{\circ}$ & 38192.373 & & & $\left\{\begin{array}{r}18924.96(30) \\
5282.58 \\
5282.52\end{array}\right.$ \\
\hline
\end{tabular}


values of the levels were arrived at by an adjustment to provide the best fit of the observed wave numbers into the scheme. The construction of this table should be fairly obvious, it being the familiar quadratic array frequently used in reporting spectral regularities. The entries comprise in each instance the observed wavelength followed by estimated intensity in parenthesis, just below the wavelength the corresponding observed wave number (alined with the combining level in the left column) and finally the calculated or adjusted wave number. The precision of the experimental observations may be judged by the agreement between observed and calculated wave numbers. There are several instances of exact agreement and no deviations greater than $0.08 \mathrm{~cm}^{-1}$. All possible transitions are observed except the faint corner satellite ${ }^{3} \mathrm{D}_{3}^{\circ}-{ }^{3} \mathrm{~F}_{2}$. The satellites in the ${ }^{3} \mathrm{~F}^{\circ}-{ }^{3} \mathrm{~F}$ multiplet are somewhat less precisely determined than the transitions on the diagonal, because they could be observed only by using the difficult experimental technique involving circulating helium in the enclosed arc in order to eliminate band structure.

The data compiled in table 3 were obtained from the measurement of records similar to the one reproduced in figure 3 , which is an ink tracing of a chart record. The records that were actually used for wavelength reductions were obtained by scanning at speeds one-half or one-fifth as fast, permitting a corresponding greater linear dispersion. In order to exhibit the weaker lines, the deflections for the more intense lines are necessarily off scale, as indicated by the blunt maxima of the strong lines. The same statement applies to figure 4 , which illustrates the extremely intense multiplet $4 p^{3} \mathrm{P}^{\circ}-3 d^{3} \mathrm{D}$. For the actual wavelength determinations of the intense lines, they are kept on the scale by an attenuator utilizing a network of resistors. A setting may be chosen to give the desired attenuation factor.

By far the strongest infrared lines and among the most intense in Ca I are included in the multiplet $4 p^{3} \mathrm{P}^{\circ}-3 d^{3} \mathrm{D}$. These are illustrated, together with $5 s^{3} \mathrm{~S}-5 p^{3} \mathrm{P}^{\circ}$ and $4 d^{1} \mathrm{D}-4 f^{1} \mathrm{~F}^{\circ}$, in figure 4 , which is traced from an actual chart record in this region. The positions of all of these lines are predictable from the values of the combining levels, given to eight figures by Wagman [15], based on interferometric measurements of wavelengths of lines, due to other combinations of these same levels, in the photographically accessible region. The calculated wave numbers given in table 2 are obtained from these precisely determined levels where possible. The $4 p{ }^{3} \mathrm{P}^{\circ}-3 d{ }^{3} \mathrm{D}$ multiplet can be observed under favorable conditions, that is high intensity and freedom from interference. These favorable factors strongly suggest the use of these lines as wavelength standards.

The agreement between observed and calculated wave numbers for lines of some multiplets in the 13000 - to $16000-\mathrm{A}$ region is not quite so good as the estimated precision of the wavelength measurements should make possible. The reason for these differences is still not fully understood, but it is suggested that the values of some of the levels may still be subject to revision. It is hardly necessary to point out that it is a prime necessity to establish a satisfactory set of standards throughout the infrared region in order that these new high-resolution techniques may be used to advantage in both atomic and molecular spectra.

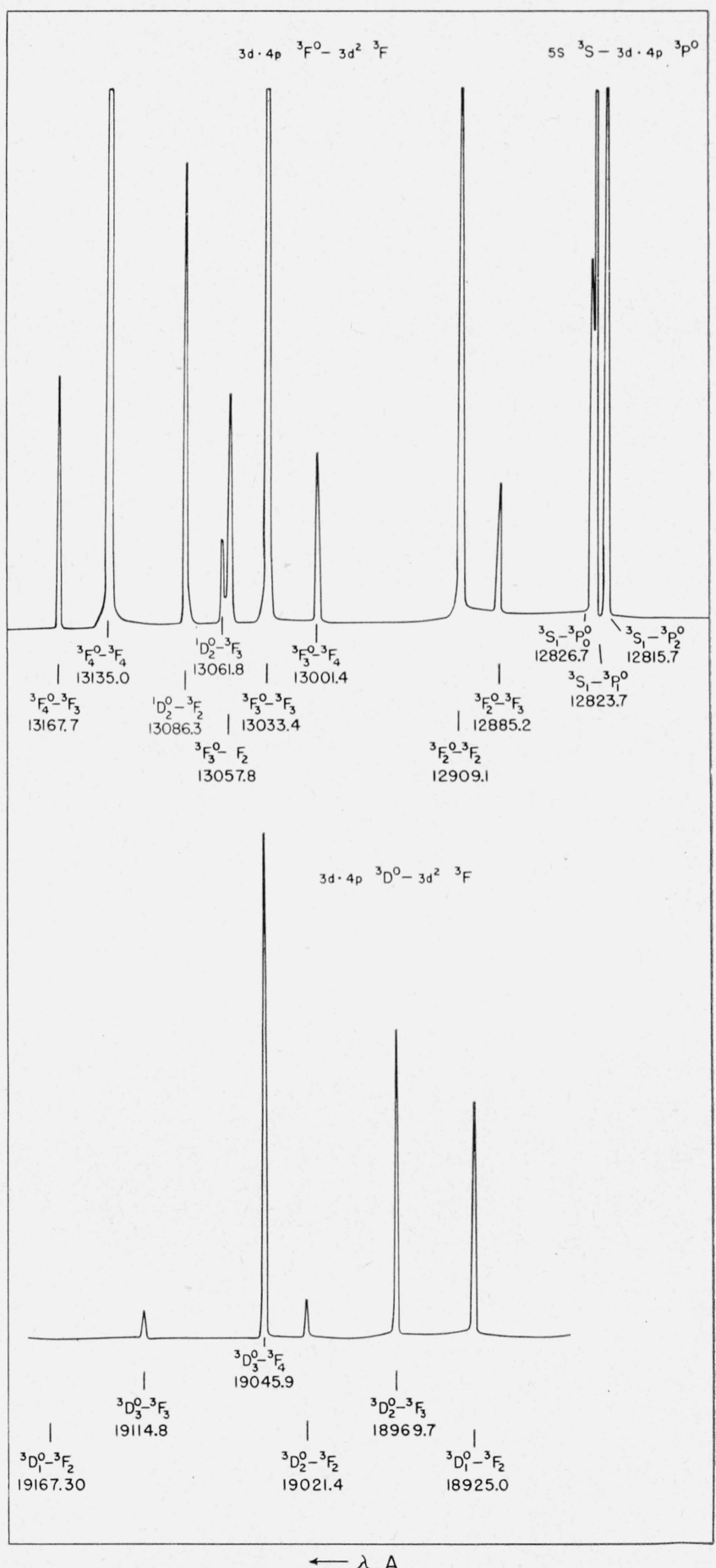

Figure 3. Multiplet combinations of $3 d^{2}{ }^{3} \mathrm{~F}$ in Ca I. 


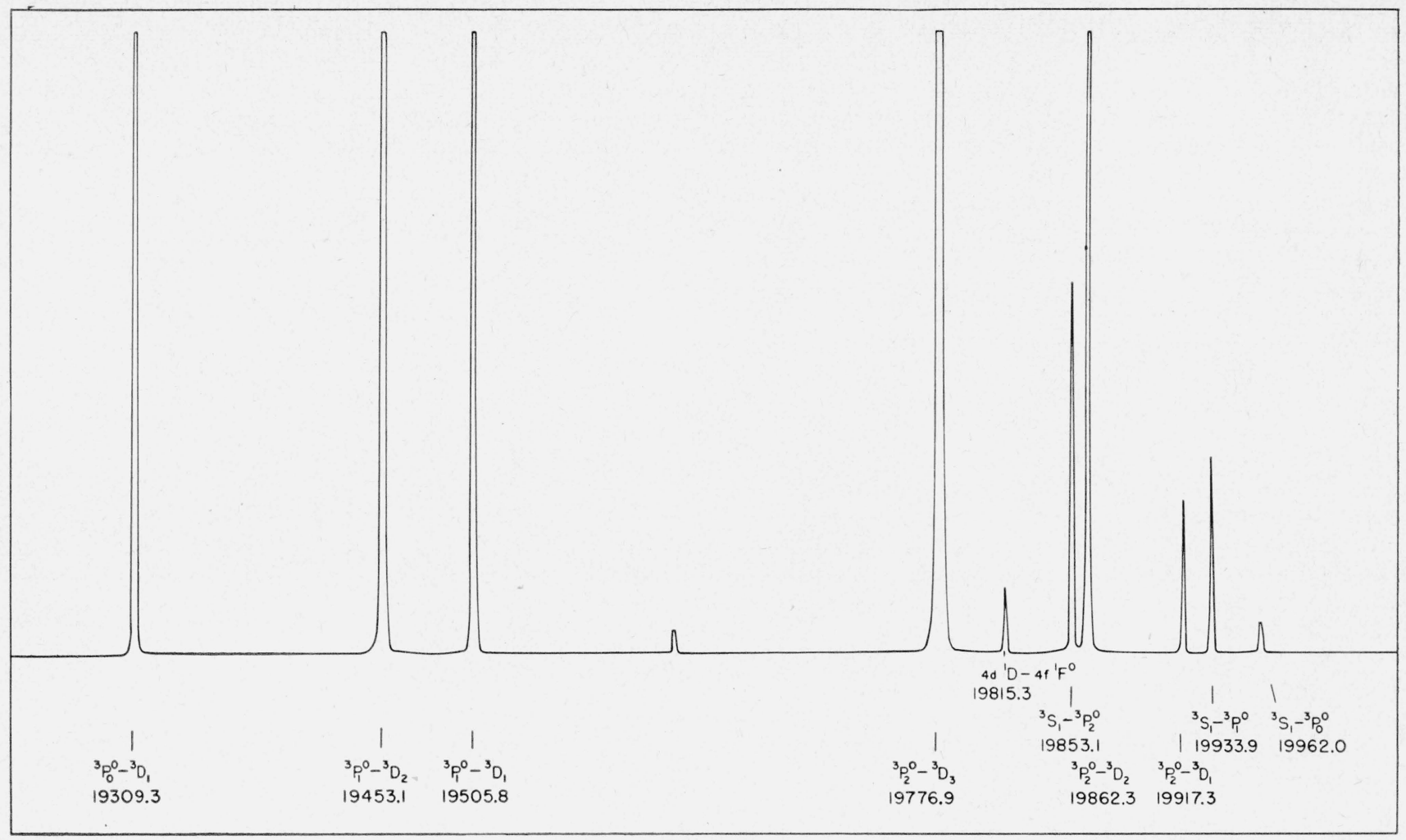

$\lambda, A \longrightarrow$

FiguRe 4. Multiplets of $\mathrm{Ca}_{\mathrm{I}}$ in the region 19300 to $20000 \mathrm{~A}$.

\section{Conclusion}

This work can hardly be said to complete the analysis of $\mathrm{Ca} \mathrm{I}$, although it has remedied the outstanding deficiency in the term array. None of the singlets either from $3 d^{2}$ or $3 d 4 d$ is known. It is probable that most of the required combinations are in the photographic infrared in the region obscured by the $(0,0) \mathrm{CN}$ band.

Further observation in the regions free of interference does not appear profitable. These experiments were undertaken as part of a larger program including the other alkaline earths. This report on calcium was prepared because of a very urgent and long-standing need for further information regarding its term structure, having in mind especially the monograph that Professor Russell has proposed. Similar observations on strontium and possibly barium are expected to yield comparable results.

The assistance of M. E. Anderson in the preparation of drawings, reproduction of records, and reduction of data, and of A. J. Cussen, in maintaining and improving the performance of the electronic accessories of the spectrometer, is gratefully acknowledged.

\section{References}

[1] Charlotte E. Moore, Atomic energy levels, NBS Circular 467 I, 242 (1949).

[2] F. Paschen and R. Götze, Seriengesetze der Linienspektren (Springer, Berlin, 1922).

[3] A. Fowler, Report on series in line spectra (Fleetway Press, London, 1922).

[4] H. N. Russell and F. A. Saunders, Astroph. J. 61, 38 (1925).

[5] F. Hund, Linienspektren und periodisches System der Elemente (Springer, Berlin, 1927).

[6] E. K. Plyer and M. A. Lamb, J. Research NBS 45, 204 (1950) RP2125.

[7] C. C. Kiess, Astroph. J. 109, 551 (1948).

[8] G. Herzberg and J. G. Phillips, Astroph. J. 108, 163 (1948).

[9] H. D. Curtis, J. Opt. Soc. Am. and Rev. Sci. Instr. 8, 697 (1924)

[10] F. Paschen, Ann. Physik. 29, 625 (1909).

[11] H. M. Randall, Ann. Physik 33, 739 (1910).

[12] O. Sandvik and B. J. Spence, Astroph. J. 62, 265 (1925).

[13] H. D. Babcock, Phys. Rev. 46, 382 (1934).

[14] L. Goldberg, O. C. Mohler, A. K. Pierce, and R. R. MeMath, Astroph. J. 111, 565 (1950).

[15] N. E. Wagman, Univ. Pittsburgh Bul. 34, 1 (1937).

Washington May 4, 1951, 